\title{
Controlling Chaos in High Dimensions
}

\author{
Celso Grebogi and Ying-Cheng Lai
}

\begin{abstract}
We review the major ideas involved in the control of chaos by considering higher dimensional dynamics. We present the Ott-Grebogi-Yorke (OGY) method of controlling chaos to achieve time periodic motion by utilizing only small feedback control. The time periodic motion results from the stabilization of unstable periodic orbits embedded in the chaotic attractor. We demonstrate that the OGY method, also applicable to high dimensions, is a particular case of the pole placement technique, and we argue that it is the one leading to shortest time to achieve control. Implementation using only a measured time series in experimental situations is described.
\end{abstract}

Index Terms - Chaos, control, pole-placement technique.

\section{INTRODUCTION}

$\mathbf{I}$ T HAS NOW been recognized that chaos occurs commonly in a large veriety of natural systems. In many applications it is often desired that chaos be avoided. One way to eliminate chaos is to make large parameter change or state alteration so as to move the system completely out of the chaotic regime. This approach, however, is not interesting as the system may not be operating in a parameter regime of interest after large change and in addition, large alteration may be costly. One thus wishes to use only small perturbation to control chaos, keeping the parameters in a close neighborhood of their nominal values. The key observation is that chaos, while signifying sensitive dependence on small changes and henceforth rendering the system state unpredictable in long time, also implies that the system's behavior can be altered by using arbitrarily small perturbations [1], [2]. Thus, quite contrary to intuition, the presence of chaos can be advantageous from the viewpoint of control, espacially because a chaotic dynamics accesses naturally different states of the system. One may wish then to design a physical, biological, or chemical experiment, or to project an industrial plant to behave in a chaotic manner to achieve a desirable performance. Furthermore, the control of chaos is also a way to manipulate natural systems that are already chaotic. The purpose of this paper is to argue that chaos may indeed be desirable since it can be manipulated by small perturbations of some accessible parameter [1], [2], or to some dynamical variable of the system [3]. We also review

Manuscript received April 10, 1997; revised June 10, 1997. This work was supported by the Department of Energy (Mathematical, Information and Computational Sciences Division, High Performance Computing and Communication Program). The work of Y.-C. Lai was supported by AFOSR under Grant F49620-96-1-0066 and by the University of Kansas. This paper was recommended by Guest Editor M. J. Ogorzałek.

C. Grebogi is with the Institut für Theoretische Physik und Astrophysik, Universität Potsdam, D-14415 Potsdam, Germany on leave from the Institute for Plasma Research, University of Maryland, College Park, MD 20742 USA.

Y.-C. Lai is with the Departments of Physics, Astronomy, and Mathematics, University of Kansas, Lawrence, KS 66045 USA.

Publisher Item Identifier S 1057-7122(97)07368-6. the Ott-Grebogi-Yorke (OGY) method of controlling chaos in higher dimensions and, when the equations of the dynamics are not known, its implementation using data analysis.

The major key ingredient for the control of chaos [1], [2] is the observation that a chaotic set, on which the trajectory of the chaotic process lives, has embedded within it a large number of unstable low-period periodic orbits [4]. Due to ergodicity, these periodic states are accessed in the course of the system's time evolution. The location of some of these periodic orbits in state space may correspond to the desired performance of the system according to some criterion. By applying small but feedback perturbations to an accessible parameter or state of the system, the state of the system can be stabilized about the desired unstable periodic orbit. Thus, the accessibility of the chaotic system to many different periodic orbits combined with its sensitivity to small perturbations allows for the control and the manipulation of the chaotic process. Specifically, the OttGrebogi-Yorke (OGY) approach is then as follows. One first determines some of the unstable low-period periodic orbits that are embedded in the chaotic set. One then examines the location and the stability of these orbits and chooses one which yields the desired system performance. Finally, one applies small control to stabilize this desired periodic orbit. All this can be done from data [1], [2] by using nonlinear time series analysis for the observation, understanding and control of the system. This is particularly important since chaotic systems are rather complicated and the detailed knowledge of the equations of the process is often unknown. The method can in principle be applied to high-dimensional chaotic systems [5]. The implementation of the method utilizes the "pole placement technique" [7] developed in the field of engineering control. We give a numerical example illustrating that.

In many engineering systems chaos is unavoidable. Controlling chaos is thus important in applications where chaos is undesirable. The OGY's approach suggests that one can actually better control chaos by taking advantage of chaos. For instance, the magnitude of the control can be made arbitrarily small and there are practically many unstable periodic states that can be stabilized to yield a desirable system performance. So far there have been many applications of the OGY's idea of chaos control to engineering problems (see [8]).

\section{Control of Chaos}

We consider the following discrete-time dynamical system,

$$
\boldsymbol{x}^{\prime}=\boldsymbol{F}(\boldsymbol{x}, p)
$$

where $\boldsymbol{x} \in \boldsymbol{R}^{N}, \boldsymbol{F}$ is a smooth vector function, and $p$ is an accessible parameter that can be externally perturbed. Continuous dynamical systems can be regarded as discrete 
maps on the Poincare surface of section. Since we conceive using only small control, we restrict $p$ to lie in some small interval

$$
|p-\bar{p}|<\delta
$$

where $\bar{p}$ is a nominal parameter value. Assuming that the dynamical system $\boldsymbol{F}(\boldsymbol{x}, \bar{p})$ possesses a chaotic attractor, our goal is to vary the parameter $p$ within the range $(\bar{p}-\delta, \bar{p}+\delta)$ in such a way that for almost all initial conditions in the basin of the chaotic attractor, the dynamics of the system converges onto a desired time periodic orbit contained in the attractor. To do this we consider a small neighborhood of size proportional and comparable to $\delta$ of the desired periodic orbit. In this neighborhood, the dynamics is approximately linear. Since linear systems are stabilizable if the perturbations obey a standard controllability condition, the chosen unstable periodic orbit is stabilized by feedback control. The ergodic nature of the chaotic dynamics guarantees that the state trajectory enters the chosen neighborhood. The stabilizing feedback control law guarantees that the trajectory is kept in the neighborhood of the desired orbit.

For simplicity we describe the method as applied to the case where the desired orbit is a fixed point (period-one orbit) of the map $\boldsymbol{F}$. Consideration of periodic orbits of period larger than one is straightforward [5]. Let $\boldsymbol{x}_{*}(p)$ be an unstable fixed point on the attractor. For values of $p$ close to $\bar{p}$ and in the neighborhood of the fixed point $\boldsymbol{x}_{*}(p)$, the map (1) can be approximated by the linear map

$$
\boldsymbol{x}^{\prime}-\boldsymbol{x}_{*}(\bar{p})=A\left[\boldsymbol{x}-\boldsymbol{x}_{*}(\bar{p})\right]+\boldsymbol{B}(p-\bar{p})
$$

where $A$ is the $N \times N$ Jacobian matrix and $\boldsymbol{B}$ is an $N$ dimensional column vector

$$
\begin{aligned}
& A=D_{x} F(x, p) \\
& B=D_{p} F(x, p) .
\end{aligned}
$$

The partial derivatives in $\boldsymbol{A}$ and $\boldsymbol{B}$ are evaluated at $\boldsymbol{x}=\boldsymbol{x}_{*}$ and $p=\bar{p}$. To calculate the parameter perturbation to $p$, we assume that it is a linear function of $\boldsymbol{x}$

$$
p-\bar{p}=-\boldsymbol{K}^{T}\left[x-x_{*}(\bar{p})\right]
$$

where the $1 \times n$ matrix $\boldsymbol{K}^{T}$ is to be determined so that the fixed point $\boldsymbol{x}_{*}$ becomes stable. Substituting (5) into (3), we obtain

$$
\boldsymbol{x}^{\prime}-\boldsymbol{x}_{*}(\bar{p})=\left(A-B \boldsymbol{K}^{T}\right)\left[\boldsymbol{x}-\boldsymbol{x}_{*}(\bar{p})\right]
$$

which shows that the fixed point will be stable upon iteration if the matrix $\left(A-B \boldsymbol{K}^{T}\right)$ is asymptotically stable; that is, if all its eigenvalues have modulus smaller than unity.

The solution to the problem of determining $\boldsymbol{K}^{T}$, such that the eigenvalues (or poles) of the matrix $\left(\boldsymbol{A}-\boldsymbol{B} \boldsymbol{K}^{T}\right)$ have specified values, is known from control systems theory as the "pole placement technique" [7]. In this context, the eigenvalues of the matrix $\left(\boldsymbol{A}-\boldsymbol{B} \boldsymbol{K}^{T}\right)$ are called the "regulator poles." The following results give a sufficient condition for a unique solution of the pole placement problem to exist, and also a method for obtaining it (Ackermann's method) [7]: (1)
The pole placement problem has a unique solution if the $N \times N$ matrix

$$
\boldsymbol{C}=\left(\boldsymbol{B}: A B \vdots A^{2} \boldsymbol{B}: \cdots \vdots A^{n-1} \boldsymbol{B}\right)
$$

is of rank $N$, where $\boldsymbol{C}$ is the controllability matrix; and (2) The solution of the pole placement problem is given by

$$
\boldsymbol{K}^{T}=\left(\alpha_{N}-a_{N}, \cdots, \alpha_{1}-a_{1}\right) \boldsymbol{T}^{-1}
$$

where $\boldsymbol{T}=\boldsymbol{C W}$ and,

$$
W=\left(\begin{array}{ccccc}
a_{N-1} & a_{N-2} & \cdots & a_{1} & 1 \\
a_{N-2} & a_{N-3} & \cdots & 1 & 0 \\
\vdots & \vdots & & \vdots & \vdots \\
a_{1} & 1 & \cdots & 0 & 0 \\
1 & 0 & \cdots & 0 & 0
\end{array}\right) .
$$

Here $\left\{a_{1}, \cdots, a_{N}\right\}$ are the coefficients of the characteristic polynominal of $\boldsymbol{A}$,

$$
|s \boldsymbol{I}-\boldsymbol{A}|=s^{N}+a_{1} s^{n-1}+\cdots+a_{N}
$$

and $\left\{\alpha_{1}, \cdots, \alpha_{N}\right\}$ are the coefficients of the desired characteristic polynomial $\left(\boldsymbol{A}-\boldsymbol{B} \boldsymbol{K}^{T}\right)$.

It should be noted that the control (5) is based on the linear (3) and therefore it is only valid in the neighborhood of the desired fixed point $\boldsymbol{x}_{*}(\bar{p})$. The size of this valid neighborhood is determined by the limitation in the size of the parameter perturbation $\delta$. Combining (2) and (5), we obtain the size of the valid neighborhood

$$
\left|K^{T}\left[\boldsymbol{x}-\boldsymbol{x}_{*}(\bar{p})\right]\right|<\delta .
$$

This defines a slab of width $2 \delta /\left|\boldsymbol{K}^{T}\right|$. We choose to activate the control according to (5) only when the trajectory falls into the slab defined by (7), and we leave the control parameter at its nominal value $\bar{p}$ when the trajectory is outside this slab. It should also be noted that the matrix $K^{T}$ can be chosen in many different ways. In principle, any choice of regulator poles inside the unit circle works [5]. The OGY method consists in setting the unstable poles equal to zero while leaving the stable ones as they are.

Since the control is turned on only when the trajectory enters the slab defined by condition (7), one has to wait for some time for this to occur if the trajectory starts from a randomly chosen initial condition in the basin of attraction of the chaotic attractor. Even then, because nonlinearity is not included in the linearized (3), the control may not be able to bring a trajectory that is already in the slab (7) to the vicinity of the periodic orbit. In this case the trajectory will leave the slab and continue to wander chaotically as if there were no control. Since the trajectory on the uncontrolled chaotic attractor is ergodic, it will eventually reenter the slab and then the controlling perturbations may be able to bring the trajectory to a close vicinity of the periodic orbit so that control is achieved. As a result, we create a stable orbit, which, for a typical initial condition, is preceded by a chaotic transient [9], [10] during which the orbit behavior is similar to the behavior of orbits on the uncontrolled chaotic attractor. The length $\tau$ of such a chaotic transient depends sensitively on the initial condition 
and it is the time for the orbit to fall in the slab (7) and be controlled. Then, in fact, $\tau$ is the time for that particular orbit to achieve control. For initial conditions randomly chosen in the basin of attraction, the probability distribution of the times to achieve control is exponential [9] for large $\tau$. The average length $\langle\tau\rangle$ is thus the average time to achieve control. It can be argued [1], [2] that $\langle\tau\rangle$ scales with $\delta$ algebraically: $\langle\tau\rangle \sim \delta^{-\gamma}$, where $\gamma>0$ is the scaling exponent that is determined by the stable and unstable eigenvalues of the desired fixed point $\boldsymbol{x}_{*}(\bar{p})$. In [5], it is shown that the OGY choice for the regulator poles yields the shortest average time to achieve control.

\section{A Higher Dimensional EXAMPLE}

As a typical higher dimensional physical system, we consider the double rotor map which is a four-dimensional map describing the time evolution of a mechanical system known as the kicked double rotor [5], [6]. The double rotor consists of two thin, massless rods as shown in Fig. 1. The first rod of length $l_{1}$ pivots about $P_{1}$ (fixed), and the second rod of length $2 l_{2}$, pivots about $P_{2}$ which moves. A mass $m_{1}$ is attached at $P_{2}$, and two masses $m_{2} / 2$ are attached to each end of the second rod. The end of the second $\operatorname{rod}\left(P_{3}\right)$ receives vertical periodic impulse kicks of period $T$ and strength $f_{0}$. The motion is in the horizontal plane so that gravity can be neglected. The double rotor is also subject to friction at $P_{1}$ and $P_{2}$ which is proportional to the angular velocity $d \theta_{1}(t) / d t$ and $d \theta_{2}(t) / d t-d \theta_{1}(t) / d t$ with proportional constants $\nu_{1}$ and $\nu_{2}$, respectively. Due to the periodic forcing, the set of differential equations describing the double rotor can be reduced to the following four-dimensional map by using the stroboscopic sectioning technique [5], [6]

$$
\left(\begin{array}{l}
\boldsymbol{X}_{n+1} \\
\boldsymbol{Y}_{n+1}
\end{array}\right)=\left(\begin{array}{c}
\boldsymbol{M} \boldsymbol{Y}_{n}+\boldsymbol{X}_{n} \\
\boldsymbol{L} \boldsymbol{Y}_{n}+\boldsymbol{G}\left(\boldsymbol{X}_{n+1}\right)
\end{array}\right)
$$

where $\boldsymbol{X}=\left(x^{1}, x^{2}\right)^{T}, \boldsymbol{Y}=\left(y^{1}, y^{2}\right)^{T}, x^{1}$ and $x^{2}$ are the angular positions of the rods at the instant of the $k$ th kick, and $y^{1}$ and $y^{2}$ are the angular velocities of the rods immediately after the $k$ th kick. $\boldsymbol{L}$ and $\boldsymbol{M}$ are constant $2 \times 2$ matrices defined by

$$
\begin{aligned}
\boldsymbol{L} & =\sum_{j=1}^{2} W_{j} e^{\lambda_{j}} T, \quad \boldsymbol{M}=\sum_{j=1}^{2} W_{j} \frac{e^{\lambda_{j} T-1}}{\lambda_{j}}, \\
W_{1} & =\left(\begin{array}{ll}
a & b \\
b & d
\end{array}\right), \quad W_{2}=\left(\begin{array}{rr}
d & -b \\
-b & a
\end{array}\right), \\
a & =\frac{1}{2}\left(1+\frac{\nu_{1}}{\Delta}\right), \quad d=\frac{1}{2}\left(1-\frac{\nu_{1}}{\Delta}\right), \quad b=-\frac{\nu_{2}}{\Delta}, \\
\lambda_{1,2} & =-\frac{1}{2}\left(\nu_{1}+\nu_{2} \pm \Delta\right), \quad \Delta=\sqrt{\nu_{1}^{2}+4 \nu_{2}^{2}} .
\end{aligned}
$$

The function $G(\boldsymbol{X})$ is given by

$$
\boldsymbol{G}(\boldsymbol{X})=\left(\begin{array}{l}
c_{1} \sin x^{1} \\
c_{2} \sin x^{2}
\end{array}\right)
$$

where $c_{1}=f_{0} l_{1} / I, c_{2}=f_{0} l_{2} / I$, and $I=\left(m_{1}+m_{2}\right) l_{1}^{2}=$ $m_{2} l_{2}^{2}$. For illustrative purposes we fix $\nu=T=I=m_{1}=$ $m_{2}=l_{2}=1$ and $l_{2}=1 / \sqrt{2}$. For $f_{0}=9.0$, the double rotor map has a chaotic attractor with two positive Lyapunov

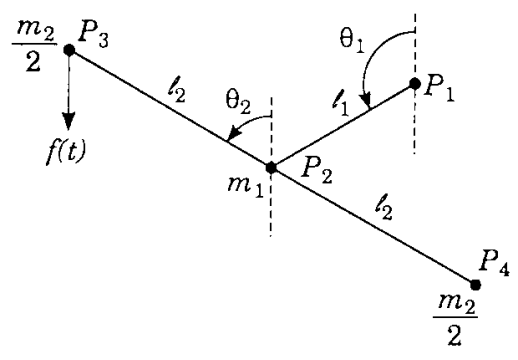

Fig. 1. The double rotor.

exponents. There are 32 unstable fixed points embedded in this chaotic attractor [5].

To apply the pole-placement technique of Section II, we choose $f_{0}$ as the control parameter so it can be varied about its nominal value $\overline{f_{0}}=9.0$. Let $\left(\boldsymbol{X}_{*}, \boldsymbol{Y}_{*}\right)$ be a fixed point to be stabilized. The quantities required in the application of the pole-placement technique are as follows:

$$
\begin{aligned}
& \boldsymbol{A}=\left(\begin{array}{cc}
\boldsymbol{I}_{2} & \boldsymbol{M} \\
\boldsymbol{H}\left(\boldsymbol{X}_{*}\right) & \boldsymbol{L}+\boldsymbol{H}\left(\boldsymbol{X}_{*}\right) \boldsymbol{M}
\end{array}\right) \\
& \boldsymbol{H}\left(\boldsymbol{X}_{*}\right)=\frac{\overline{f_{0}}}{I}\left(\begin{array}{cc}
l_{1} \cos x_{*}^{1} & 0 \\
0 & l_{2} \cos x_{*}^{2}
\end{array}\right) \text {, } \\
& B^{T}=\left(0,0, l_{1} \sin x_{*}^{1} / I, l_{2} \sin x_{*}^{2} / I\right), \\
& \boldsymbol{C}=\left(\boldsymbol{B}: A B: A^{2} B: \cdots \vdots A^{n-1} B\right), \quad T=C W, \\
& \boldsymbol{W}=\left(\begin{array}{cccc}
a_{3} & a_{2} & a_{1} & 1 \\
a_{2} & a_{1} & 1 & 0 \\
a_{1} & 1 & 0 & 0 \\
1 & 0 & 0 & 0
\end{array}\right), \\
& \boldsymbol{K}^{T}=\left(\alpha_{4}-a_{4}, \alpha_{3}-a_{3}, \alpha_{2}-a_{2}, \alpha_{1}-a_{1}\right) T^{-1} \text {. }
\end{aligned}
$$

Fig. 2(a) and (b) show how the method works to stabilize different unstable fixed points embedded in the chaotic attractor. Control of the first fixed point was turned on when the trajectory enters the its slab defined by (7) with switches to control other fixed points occurring at later times. In the figures, the state variables $x^{1}$ and $x^{2}$ of an orbit are plotted. The times at which the control is switched from stabilizing one fixed point to stabilizing another are labeled by the arrows in the figures. The magnitude of the parameter perturbation is chosen as $\delta=1.0$, which is roughly $10 \%$ of the nominal $f_{0}$ value. A smaller $\delta$ would increase the average time to achieve control. The figures clearly illustrate the flexibility offered by the method in controlling different periodic motions embedded in the attractor.

\section{USE OF DELAY COORDINATES}

In most experimental situations a detailed knowledge of the system's equations is not known. In that case, one usually obtains a time series by measuring a single scalar state variable, say $u(t)$, and then using delay coordinates [11] to represent the evolution of the system state and to extract the quantities necessary for the control. A delay-coordinate vector in the $m$-dimensional embedding space can be formed as follows:

$\boldsymbol{x}(t)=\left(u(t), u\left(t-t_{D}\right), u\left(t-2 t_{D}\right), \cdots, u\left(t-(m-1) t_{D}\right)\right)$, 


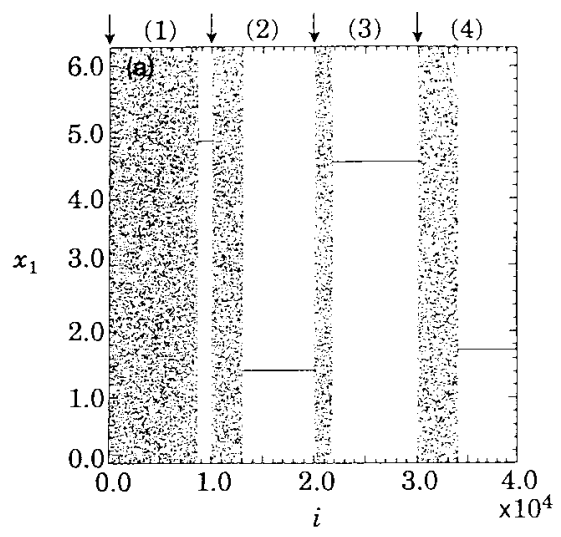

(a)

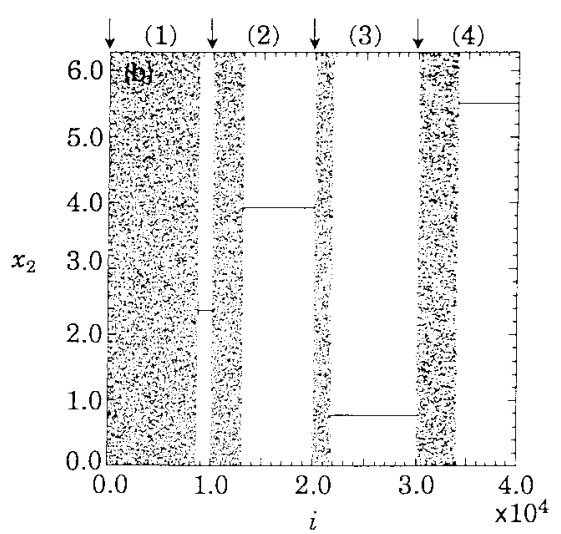

(b)

Fig. 2. (a), (b) Double rotor map: successive control of unstable fixed points embedded in the chaotic attractor. The arrows indicate the times of switching.

where $t$ is the continuous time variable, and $t_{D}$ is some conveniently chosen delay time. The embedding theorem [11], [12] guarantees that for $m \geq 2 N$, where $N$ is the phase-space dimension of the system, the vector $\boldsymbol{x}$ is generically a global one-to-one representation of the system state. Since, in the control problem, we only require $\boldsymbol{x}$ to be one-to-one in the small region near the periodic orbit, the requirement for the embedding dimension is actually $m=N-1$ [1]. To obtain a map, one can take a Poincaré surface of section. For the often encountered case of periodically driven systems, one can define a "stroboscopic surface of section" by sampling the state at discrete time $t_{n}=n T+t_{0}$, where $T$ is the driving period. In this case the discrete state variable is $\boldsymbol{x}_{n}=\boldsymbol{x}\left(t_{n}\right)$.

As pointed out in [13], in the presence of parameter variation, delay coordinates lead to a map of a different form from (1). For example, in the periodically forced case, since the components of $x_{n}$ are $u\left(t-i t_{D}\right)$ for $i=0,1, \cdots,(m-1)$, the vector $\boldsymbol{x}_{n+1}$ depends not only on $p_{n}$, but also on all previous values of the parameter that are in effect during the time interval $\left(t_{n}-(m-1) t_{D}\right) \leq t \leq t_{n}$. In particular, let $r$ be the smallest integer such that $m t_{D}<r T$. Then the relevant map is in general of the form

$$
\boldsymbol{x}_{n+1}=\boldsymbol{G}\left(\boldsymbol{x}_{n}, p_{n}, p_{n-1}, \cdots, p_{n-r}\right) .
$$

We now discuss how the OGY method can be applied to the case of delay coordinates. For simplicity we consider $r=1$.
In this case, we have,

$$
x_{n+1}=G\left(\boldsymbol{x}_{n}, p_{n}, p_{n-1}\right)
$$

Linearizing as in (3) and again restricting our attention to the case of a fixed point, we have

$$
\begin{aligned}
\boldsymbol{x}_{n+1}-\boldsymbol{x}_{*}(\bar{p})= & A\left[\boldsymbol{x}_{n}-\boldsymbol{x}_{*}(\bar{p})\right]+\boldsymbol{B}_{a}\left(p_{n}-\bar{p}\right) \\
& +\boldsymbol{B}_{b}\left(p_{n-1}-\bar{p}\right)
\end{aligned}
$$

where $\boldsymbol{A}=\boldsymbol{D}_{\boldsymbol{x}} \boldsymbol{G}\left(\boldsymbol{x}, p, p^{\prime}\right), \boldsymbol{B}_{a}=\boldsymbol{D}_{p} \boldsymbol{G}\left(\boldsymbol{x}, p, p^{\prime}\right), \boldsymbol{B}_{b}=$ $\boldsymbol{D}_{p^{\prime}} \boldsymbol{G}\left(\boldsymbol{x}, p, p^{\prime}\right)$, and all partial derivatives in $\boldsymbol{A}, \boldsymbol{B}_{a}$, and $\boldsymbol{B}_{b}$ are evaluated at $\boldsymbol{x}=\boldsymbol{x}_{*}(\bar{p})$ and $p=\bar{p}=p^{\prime}$. One can now define a new state variable with one extra component by

$$
\overline{\boldsymbol{x}}_{n+1}=\left(\begin{array}{c}
\boldsymbol{x}_{n+1} \\
p_{n}
\end{array}\right)
$$

and introduce the linear control law

$$
p_{n}-\bar{p}=-\boldsymbol{K}^{T}\left[\boldsymbol{x}_{n}-\boldsymbol{x}_{*}(\bar{p})\right]-k\left(p_{n-1}-\bar{p}\right) .
$$

Combining (14) and (16), we obtain

$$
\overline{\boldsymbol{x}}_{n+1}-\overline{\boldsymbol{x}}_{*}(\bar{p})=\left(\overline{\boldsymbol{A}}-\overline{\boldsymbol{B K}}^{T}\right)\left[\overline{\boldsymbol{x}}-\overline{\boldsymbol{x}}_{*}(\bar{p})\right]
$$

where

$$
\begin{aligned}
\overline{\boldsymbol{x}}_{*}(\bar{p}) & =\left(\begin{array}{c}
\boldsymbol{x}_{*}(\bar{p}) \\
\bar{p}
\end{array}\right), \quad \bar{A}=\left(\begin{array}{cc}
\boldsymbol{A} & \boldsymbol{B}_{b} \\
\mathbf{0} & 0
\end{array}\right), \\
\overline{\boldsymbol{B}} & =\left(\begin{array}{c}
\boldsymbol{B}_{a} \\
1
\end{array}\right), \quad \overline{\boldsymbol{K}}=\left(\begin{array}{c}
\boldsymbol{K} \\
k
\end{array}\right) .
\end{aligned}
$$

Since (17) is now of the same form as (6), the method of Section II can be applied. A similar result holds for any $r>1$. Although the explicit form for the function $\boldsymbol{G}\left(\boldsymbol{x}_{n}, p_{n}, p_{n-1}\right)$ is not known, the quantities required for computing the parameter perturbations in (16) can usually be extracted directly from the measurement [14].

\section{COMMENTS}

An important issue in controlling chaos using small perturbations is the effect of noise. Unbounded noise can in general kick the controlled trajectory out of the neighborhood of the chosen periodic orbit where the control is activated. When this occurs, the trajectory wanders chaotically over the attractor until it falls in the controlled region (7) again. Thus there are epochs where the orbit is kept near the desired orbit interspersed with epochs wherein the orbit wanders chaotically far from the desired orbit. If the latter are, on average, relatively much shorter than the former, then one might still regard the control as being effective.

The transient phase where the orbit wanders chaotically before locking into a controlled orbit can be greatly shortened by applying a "targeting" technique [15], [16] so that a trajectory can be rapidly brought to a target region on the attractor by using small control perturbations. The idea is that, since chaotic systems are exponentially sensitive to perturbations, careful choice of even small control perturbations can, after some time, have a large effect on the trajectory location and this can be used to guide it to a target on the attractor. Thus the time to achieve control can, in principle, be greatly reduced 
by properly applying small controls when the orbit is far from the neighborhood of the desired periodic orbit.

In this paper we have considered the case where there is only a single control parameter available for adjustment. While generically a single parameter is sufficient for stabilization of a desired periodic orbit, there may be some advantage to utilizing several control variables [17]. Therefore, the single control parameter $p$ becomes a vector. In particular, the added freedom in having several control parameters might allow better means of choosing the control so as to minimize the time to achieve control, as well as the effects of noise.

We emphasize that full knowledge of the system dynamics is not necessary in order to apply the OGY idea [1], [2]. In particular, we only require the location of the desired periodic orbit, the linearized dynamics about the periodic orbit, and the dependence of the location of the periodic orbit on small variation of the control parameter. Delay-coordinate embedding has been successfully utilized in experimental studies to extract such information purely from observation of experimental chaotic orbits on the attractor without any $a$ priori knowledge of the equations of the system, and such information has been utilized for the control of chaos [14].

Finally, we restate that the OGY idea of controlling chaos gives flexibility. By switching the small control, one can switch the time asymptotic behavior from one periodic orbit to another in chosen locations in the state space. In some applications, where the flexibility offered by the ability to do such switching is desirable, it is advantageous to design the system so that it is chaotic. In other situations, where one is presented with a chaotic system, the method may allow one to eliminate chaos and achieve greatly improved behavior with small perturbations and relatively low cost. The OGY ideas can also be used to stabilize a desired chaotic trajectory, which has potential applications to problems such as synchronization of chaotic systems [18], conversion of transient chaos into sustained chaos [19], and selection of a desired chaotic phase [20], [21]. One can also encode any desired symbol sequence in the chaotic dynamics and hence use these ideas for communicating with chaos [3], [22], [23].

\section{REFERENCES}

[1] E. Ott, C. Grebogi, and J. A. Yorke, "Controlling chaos," Phys. Rev. Lett., vol. 64, pp. 1196-1199, 1990.

[2] , "Controlling chaotic dynamical systems," pp. 153-172 in CHAOS/XAOC, Soviet-Amer. Perspective on Nonlinear Science, D. Campbell, Ed. New York: Amer. Inst. Phys., 1990.
[3] S. Hayes, C. Grebogi, and E. Ott, "Communicating with chaos," Phys. Rev. Lett., vol. 70, pp. 3031-3034, 1993.

[4] C. Grebogi, E. Ott, and J. A. Yorke, "Unstable periodic orbits and the dimension of chaotic attractors," Phys. Rev. A, vol. 37, pp. 1711-1724, 1988.

[5] F. J. Romeiras, C. Grebogi, E. Ott, and W. P. Dayawansa, "Controlling chaotic dynamical systems," Physica D, vol. 58, pp. 165-192, 1992.

[6] C. Grebogi, E. Kostelich, E. Ott, and J. A. Yorke, "Multidimensional intertwined basin boundaries: basin structure of the kicked double rotor," Physica D, vol. 25, pp. 347-360, 1987.

[7] K. Ogata, Modern Control Engineering, 2nd Ed. Englewood Cliffs, NJ: Prentice-Hall, 1990

[8] J. M. T. Thompson and S. R. Bishop, Nonlinearity and Chaos in Engineering Dynamics. New York: Wiley, 1994.

[9] C. Grebogi, E. Ott, and J. A. Yorke, "Crises, sudden changes in chaotic attractors and chaotic transients," Physica D, vol. 7, pp. 181-200, 1983.

[10] C. Grebogi, E. Ott, F. J. Romeiras, and J. A. Yorke, "Critical exponent for crisis-induced intermittency," Phys. Rev. A, vol. 36, pp. 5365-5380, 1987.

[11] F. Takens, "Detecting strange attractors in turbulence," pp. 366-381 in Dynamical Systems and Turbulence (Lecture Notes in Mathematics, vol. 898). D. A. Rand and L.-S. Young, Eds. New York: Springer, 1980.

[12] T. Sauer, J. A. Yorke, and M. Casdagli, "Embedology," J. Stat. Phys., vol. 65 , pp. $579-616,1991$.

[13] U. Dressler and G. Nitsche, "Controlling chaos using time delay coordinates," Phys. Rev. Lett., vol. 68, pp. 1-4, 1992.

[14] W. L. Ditto, S. N. Rauseo, and M. L. Spano, "Experimental control of chaos," Phys. Rev. Lett., vol. 65, pp. 3211-3214, 1990.

[15] T. Shinbrot, E. Ott, C. Grebogi, and J. A. Yorke, "Using chaos to direct trajectories to targets," Phys. Rev. Lett., vol. 65, pp. 3215-3218, 1990

[16] E. J. Kostelich, C. Grebogi, E. Ott, and J. A. Yorke, "High dimensional targeting," Phys. Rev. E, vol. 47, pp. 305-310, 1993.

[17] E. Barreto and C. Grebogi, "Multiparameter control of chaos," Phys. Rev. E, vol. 52, pp. 3553-3557, 1995.

[18] Y.-C. Lai and C. Grebogi, "Synchronization of chaotic trajectories using control," Phys. Rev. E, vol. 47, pp. 2357-2360, 1993.

[19] - "Converting transient chaos into sustained chaos by feedback control," Phys. Rev. E, vol. 49, pp. 1094-1099, 1994.

[20] Y. Nagai and Y.-C. Lai, "Selection of desirable chaotic phase using small feedback control," Phys. Rev. E, vol. 51, pp. 3842-3848, 1995.

[21] M. G. Rosenblum, A. S. Pikovsky, and J. Kurths, "Phase synchronization of chaotic oscillators," Phys. Rev. Lett., vol. 76, pp. 1804-1807, 1996.

[22] S. Hayes, C. Grebogi, E. Ott, and A. Mark, "Experimental control of chaos for communication," Phys. Rev. Lett., vol. 73, pp. 1781-1784, 1994.

[23] E. Rosa, S. Hayes, and C. Grebogi, "Noise filtering in communicating with chaos," Phys. Rev. Lett., vol. 78, pp. 1247-1250, 1997.

Celso Grebogi is a Professor of Mathematics and Distinguished Faculty Research Fellow with the University of Maryland, College Park.

Prof. Gregogi is a Fellow of the American Physical Society and has been awarded the Senior Humboldt Prize, the Toshiba Chair, and the Fulbright Fellowship.

Ying-Cheng Lai is an Assistant Professor of physics and mathematics with the University of Kansas, Lawrence.

Dr. Lai is the recipient of an NSF Faculty Career Award. 